\title{
Relativistic effects on dust grain charging in non-equilibrium dusty plasma with relativistic Tsallis distribution
}

Cite as: Phys. Plasmas 26, 113704 (2019); https://doi.org/10.1063/1.5123657

Submitted: 12 August 2019. Accepted: 15 October 2019 . Published Online: 20 November 2019

Masoud Taherimoghadam, Iman Motie, Ali Bakhshayeshi, and Taghi Mirzaye

\section{ARTICLES YOU MAY BE INTERESTED IN}

On the generalized formulation of Debye shielding in plasmas

Physics of Plasmas 26, 050701 (2019); https://doi.org/10.1063/1.5091949

On stretching of plasma parameters and related open issues for the study of dust-ionacoustic and dust-acoustic shock waves in dusty plasmas

Physics of Plasmas 26, 084501 (2019); https://doi.org/10.1063/1.5112824

Three-dimensional plasmoid-mediated reconnection and the effect of toroidal guide field in simulations of coaxial helicity injection

Physics of Plasmas 26, 092502 (2019); https://doi.org/10.1063/1.5098482

\begin{tabular}{|l|l|l|}
\hline AVS Quantum Science & $\begin{array}{l}\text { A high impact interdisciplinary } \\
\text { journal for ALL quantum science }\end{array}$ \\
AIP ACCEPING SUBMISSIONS \\
\hline Autshing
\end{tabular}




\title{
Relativistic effects on dust grain charging in non-equilibrium dusty plasma with relativistic Tsallis distribution
}

\author{
Cite as: Phys. Plasmas 26, 113704 (2019); doi: 10.1063/1.5123657 \\ Submitted: 12 August $2019 \cdot$ Accepted: 15 October 2019 • \\ Published Online: 20 November 2019
}

Masoud Taherimoghadam, ${ }^{\text {a) }}$ Iman Motie, ${ }^{\text {b) }}$ Ali Bakhshayeshi, ${ }^{\mathrm{c})}$ and Taghi Mirzaye ${ }^{\mathrm{d})}$

\author{
AFFILIATIONS \\ a)taheri@bojnourdiau.ac.ir \\ b)imanmoti@gmail.com \\ c)bakhshayeshi.ali@gmail.com \\ d) tmirzaye@gmail.com
}

Department of Physics, Mashhad Branch, Islamic Azad University, Mashhad, Iran

\begin{abstract}
The relativistic effects on the dust grain charging are investigated by calculating the relativistic cross section by the orbit-limited motion (OLM) theory and relativistic Tsallis distribution function for currents carried by ions and electrons in nonequilibrium plasma. The dust grain electrical potential equation is derived in the dust grain charging process, and this nonlinear equation is solved by the numerical method. We indicate that the behavior of nonextensivity is in contrast to ions and electrons, and it is found that the density of species in plasma has the most influence on the degree of nonextensivity. We show that the degree of nonextensivity of the electron and the ion has significant effects on the relationship between dust grain electrical potential and dust density. As another result, it is indicated that by increasing the dust density, the dust particles will be seen as a component from ordinary multi-ionic plasma that shows collective behavior. By comparing the results in relativistic and nonrelativistic regimes, it is indicated that in the relativistic regime, the density of dust is limited, and the electrical potential of dust grains also varies, whereas in the nonrelativistic regime, the dust density is not limited, and the dust grain electrical potential tends to be constant in small amounts of dust density.
\end{abstract}

Published under license by AIP Publishing. https://doi.org/10.1063/1.5123657

\section{INTRODUCTION}

There are many different processes in the physical, chemical, biological, technical, and interdisciplinary fields. Understanding their nature is very important. In order to understand the physics of these systems, we just know the raw details of all states, statistical mechanics, and distribution functions. ${ }^{1,2}$ Some distributions associated with complex systems have been modeled within the framework of nonextensive statistics. ${ }^{2,3}$ Theoretically, dusty plasma is a complex system that shows nonextensivity effects in numerous aspects, and thus, it is rarely in equilibrium. ${ }^{4-6}$ Boltzmann-Gibbs (BG) statistical mechanics is suitable for describing classical equilibrium systems. ${ }^{7,8}$ In contrast, Tsallis statistical mechanics, based on a nonextensive formulation of entropy, has offered a theoretical basis for describing and analyzing complex systems out of equilibrium. ${ }^{5,9}$ Nonextensive entropy is a generalization of Boltzmann-Gibbs entropy by Tsallis in 1988. ${ }^{2,3}$ The Tsallis distribution can be considered as the more appropriate basis of a theoretical framework where long-range interactions, long-range microscopic memories, and/or fractal space-time constraints are present such as plasmas and gravitational systems. ${ }^{2,9}$ Boltzmann-Gibbs statistical mechanics discusses systems that have short-range interactions and large dimensions, but the statistical mechanics of Tsallis are related to small systems with long-range and complex interactions. Meanwhile, the $\mathrm{q}$ index is the boundary between Boltzmann-Gibbs and Tsallis. This means that Boltzmann-Gibbs mechanics will appear in $\mathrm{q}=1$, and we will have the statistical mechanics of the Tsallis for $\mathrm{q} \neq 1$. $^{2}$ At present, the Tsallis distribution and related nonextensive statistical mechanics have been successfully implemented in a large number of complex nonextensive systems, e.g., biological, economics, dissipative optical lattices, trapped ions, high-energy collisions at LHC/CERN, two-dimensional Euler and drift turbulence in a pure electron plasma column, velocity function of galaxy clusters, the solar neutrino problem, stellar polytropes, anomalous diffusion of a quark in a quarkgluon plasma, flux of cosmic rays on Earth, collisions involving heavy nuclei, in D-He fusion plasma experiments, etc. ${ }^{10-18}$ In theoretical and 
empirical efforts, the topics of the nonextensive statistical distribution functions in active fields on physics research, especially plasma and dusty plasma, are addressed. In an empirical study, Silva et al. ${ }^{19}$ demonstrated experimentally that particle distribution measurements are well according to the non-Maxwellian distribution function. In another research work, Caruso et al. ${ }^{20}$ analyzed the earthquake using the q-distribution function, called quasi-Gaussian distribution function, and showed that the results correspond to the experimental data. It is shown by Plastino and Plastino ${ }^{21}$ that Tsallis entropy is suitable to find sensible distribution functions for stellar polytropes, while that of Boltzmann yields unphysical distributions. In a research paper, Wong et al. ${ }^{22}$ found that the results of empirical work and theoretical studies based on the Maxwell distribution differ by about 70 percent, and thus, the B-G statistics cannot correctly predict the empirical results, while in the research work of Lima et al., ${ }^{23}$ utilized Tsallis statistics is fitted well with empirical data. They used the Tsallis distribution function for two-dimensional Euler and drift turbulence in a pure-electron plasma column, and in another research work, Boghosian ${ }^{24}$ provided a thermodynamic description of it also. However, all the abovementioned works have been constrained in the nonrelativistic plasmas, but in some situations such as in the Van Allen radiation belts, in interstellar medium, space plasma, in earth magnetosphere, where the velocity of ion and electron species becomes approximately similar to the speed of light, the consideration of relativistic behavior of plasma has significant role in modifying the nonlinear structures. ${ }^{25-31}$ In addition, in the Tsallis statistic, another important issue is determining the amount of $\mathrm{q}$ that is the nonextensivity degree of the system and one of the open issues in Tsallis statistics. Lina et al. ${ }^{32}$ in a research work have studied the relativistic nonextensive distribution function on a relativistic gas in an electromagnetic field, and they determined the nonextensivity of the system. Another research effort by Hamity and Barraco $^{33}$ worked on the Tsallis thermodynamics for the cosmic blackbody radiation in the Robertson-Walker model. They showed that on a highly localized and local scale, the temperature of the cosmic blackbody radiation was independent of $\mathrm{q}$, while at the large scale by Plastino et al., ${ }^{34}$ the dependence of $\mathrm{q}$ has been shown. Moreover, Tsallis et al. ${ }^{35}$ have shown in another study that the black-body spectrum is dependent on parameter q. In a study, Lavagno et al. ${ }^{36}$ showed that the observational data of the velocity distribution of galaxy clusters are according to a nonextensive statistical distribution. Also, they showed that solar neutrino fluxes can be understood using nonextensive statistics for the central core plasma and values of $\mathrm{q}$ slightly below one: $\mathrm{q}=0.997$ (ion plasma) and $\mathrm{q}=0.976$ (electron plasma). Moreover, Tsallis ${ }^{37}$ in a research paper showed that the nonextensivity degree for the sun with a value of $\mathrm{q}=0.24$ is in good harmony.

In this work, we study the process of dust grain charging and calculate the electrical potential function of a dust grain by considering Orbital-Limited Motion Theory (OLM). This theory (OLM) is the most commonly used theory of dust grain charging and was initially proposed by Langmuir and $\mathrm{Smith}^{38}$ and completed in the 1960s. ${ }^{16,39}$ Although this theory uses simple concepts such as angular motion and collision cross section, it predicts the dust grain electrical potential with acceptable accuracy for a wide range of grain sizes. ${ }^{40-44}$ We investigate the effects of the temperature, gas of plasma, and also the degree of nonextensivity $\left(\mathrm{q}_{\mathrm{e}}\right.$ and $\left.\mathrm{q}_{\mathrm{i}}\right)$ in the relativistic regime on the dust grain charging process and as a result obtain dust grain electrical potential using a kinetic model and quasi-neutrality condition of plasma. It is assumed that the dust grain potential is negative because the mass of the ion is much larger than the mass of the electron, and thus, the electron reaches faster than the ion on the surface of a dust grain. The relativistic Tsallis distribution for dust grain charging currents in plasma is considered. It has been realized that the particle velocity distribution function can change from nonrelativistic to relativistic behavior in some space and laboratory plasmas. In some conditions, such as the Van Allen radiation belts, interstellar plasma, on earth's magnetosphere, where the particle velocities are approximately close to the speed of light, consideration of relativistic behavior in plasma plays an important role especially in correction of complex nonlinear plasma systems and structures. In solar physics and around Earth's orbit, it is observed that electron distribution functions are isotropic approximately from thermal energies to modestly relativistic energies. ${ }^{18,27}$ In this area, some of the particle velocities can be about $0.08<\mathrm{v} / \mathrm{c}<0.8$, and thus, plasma can be defined in the relativistic regime. ${ }^{27}$ Recent findings by the HESS experiment reveal supernova RX J1713.7-3946 as the source of the $\mathrm{TeV}$ cosmic rays. ${ }^{26}$ In this way, we calculated the currents carried by electrons and ions on the dust grain charging process, and thus generally, the results are compared by nonrelativistic Tsallis distribution. ${ }^{45}$

This work is organized as follows: In Sec. II, by relativistic Tsallis distribution function, the basic formula OLM theory and the relativistic cross section for dust grain charging are obtained. Moreover, the relativistic electron and ion currents on a dust grain for calculating the dust grain electrical potential are obtained. In Sec. III, the numerical solution of the nonlinear equation is presented, and results are discussed. The role of relativistic effects on the dust grain electrical potential by changing parameters such as the degree of nonextensivity $q$ and temperature of electron to ion and gas plasma is investigated. Finally, a summary and conclusions are given in Sec. IV.

\section{BASIC EQUATIONS AND CHARGING PROCESSES}

In order to consider the dust grain charging process, it is necessary to start from the collision cross section between the dust grain with electron and ion currents in relativistic dusty plasma. The relativistic nonmagnetic plasma contains electrons, ions, and dust particles immersed in it as a spherical dust grain of radius $r_{d}$ and charge $q_{d}$ that $\varphi_{\mathrm{d}}=\mathrm{q}_{\mathrm{d}} / \mathrm{r}_{\mathrm{d}}$ is the plasma potential in conditions $\mathrm{r}_{\mathrm{d}} \ll \lambda_{\mathrm{D}}<$ a where $\lambda_{\mathrm{D}}$ is the Debye length and $\mathrm{a}$ is the dust grain average distance (for example in collapse of a star or supernova explosions where relativistic conditions prevail, a plasma jet injects into the ambient medium, e.g., in the interstellar medium or the stellar wind of the progenitor star, if the temperature and density of the electrons are 100 Gev and $10^{19} \mathrm{~cm}^{-3}$, respectively, the Debye length is about $1 \mathrm{~mm}$, assuming a dust particle of $1 \mu \mathrm{m}$ with a density of $10^{-12} \mathrm{~cm}^{-3}$ and then $\mathrm{a}=10^{4} \mathrm{~cm}$, and thus, the dust particles will be isolated $\left.{ }^{26,46}\right)$. The plasma particle path changes when it enters in the Debye sphere and feels the influence of the dust grain charge due to the electrostatic force. The relativistic cross section for charging collisions with the impact parameter $b_{j}$ between the dust grain and the plasma particle $j$ is assumed as $\sigma=\pi b_{j}^{2}$ where $j$ is the electron or ion $(j=e$ or $i)$. Assuming that $\mathrm{v}_{\mathrm{j}}$ and $\mathrm{v}_{\mathrm{gj}}$ are plasma particle velocity before and after grazing collision with the dust grain, then the conservation of angular momentum is given in the following equation: 


$$
M_{j} v_{j} b_{j}=M_{j} v_{\mathrm{gj}} r_{d},
$$

where $M_{j}$ is relativistic mass. The energy conservation is $E_{1}=E_{2}$, where $E_{1}$ and $E_{2}$ are plasma particle energy before and after grazing collision with the dust grain, respectively. Thus, in the ultrarelativistic regime and the local framework, the conservation of energy is

$$
P_{j_{1}} c=P_{j_{2}} c+\frac{q_{j} q_{d}}{r_{d}},
$$

where $P_{j}=M_{j} v_{j}$ is the linear momentum, the indices 1 and 2 are related to before and after grazing collision with the dust grain, and $\mathrm{q}_{j}$ is the charge of the plasma particle. Due to the conservation of momentum and energy, the relativistic cross section is

$$
\begin{gathered}
\sigma=\pi r_{d}^{2}\left(1-\frac{\sqrt{1-\beta_{j}^{2}}}{\beta_{j}} u_{j}\right)^{2}, \\
u_{j}=\frac{q_{j} \varphi_{d}}{m_{j} c^{2}},
\end{gathered}
$$

where $u_{j}$ is the ratio of potential energy to rest energy, $\beta_{\mathrm{j}}=\mathrm{v}_{\mathrm{j}} / \mathrm{c}$ is the relativistic factor, $v_{j}$ is the speed of the plasma particle before its grazing collision with the dust grain, $\mathrm{c}$ is the velocity of light, and $\mathrm{m}_{\mathrm{j}}$ and $\mathrm{q}_{\mathrm{j}}$ are the rest mass and charge of plasma particle, respectively.

The Tsallis distribution description for a relativistic plasma can be written as ${ }^{31,47}$

$$
f_{q_{j}}\left(v_{j}\right)=A_{q_{j}}\left[1-\left(q_{j}-1\right) \frac{\varepsilon_{j}}{k_{B} T_{j}}\right]^{\frac{1}{q_{j}-1}},
$$

where the normalization constant is

$$
A_{q_{j}}=\frac{n_{0 j}}{4 \pi\left(m_{j} c\right)^{3} \lambda_{j}}, \quad \frac{2}{3}<q_{j}<1+1 / \alpha_{j},
$$

in which

$$
\lambda_{j}=\frac{1}{c^{3}} \int_{0}^{v_{j}}\left[1-\left(q_{j}-1\right) \frac{\varepsilon_{j}}{k_{B} T_{j}}\right]^{\frac{1}{q_{j}-1}} v_{j}^{2} d v_{j},
$$

where $\mathrm{q}_{\mathrm{j}}$ is the nonextensive parameter, $\varepsilon=\gamma \mathrm{m}_{\mathrm{j}} \mathrm{c}^{2}$ is the relativistic energy, $\gamma=\left(1-\beta_{j}^{2}\right)^{-1 / 2}$ is the relativistic factors, $\alpha_{j}=m_{j} c^{2} / k_{B} T_{j}$ is the temperature parameter, $k_{B}$ is the Boltzmann constant, and $T_{j}$ and $n_{0 j}$ denote, respectively, the temperature and density of the plasma particle. Let us stress when $q_{j} \rightarrow 1$, the Boltzmann-Gibbs result is recovered, and for $\mathrm{q}_{j} \neq 1$, the distribution and the basic property of entropy are nonextensive. For $\mathrm{q}_{j}<2 / 3$, the $\mathrm{q}$-distribution is unnormalizable, and the velocity of the particles can take any value for $2 / 3<\mathrm{q}_{j}<1$. Furthermore in $\mathrm{q}_{j} \geq 1$, the distribution function exhibits a cutoff on the maximum value for the velocity of the particles

$$
v_{j, \max }=\sqrt{\left[1 / \alpha_{j}\left(q_{j}-1\right)\right]^{2}-1}
$$

that shows the real value of cutoff exists for $q_{j} \leq 1+1 / \alpha_{j}{ }^{31}$ Now, it is important to know what are the dust grain charging currents in plasma with the relativistic Tsallis distribution function. Based on the OLM theory, $I_{j}$ results from plasma particles with the charge $q_{j}$ and the relativistic velocity distribution $\mathrm{f}_{\mathrm{qj}}\left(\mathrm{v}_{\mathrm{j}}\right)^{48}$

$$
I_{j}=q_{j} \int_{v_{j}^{\min }}^{c} v_{j} \sigma_{j}^{d} f_{q_{j}}\left(v_{j}\right) d v_{j} .
$$

It is important in the relativistic regime that $\mathrm{v}_{\mathrm{j}}^{\mathrm{min}} \leq \mathrm{v}_{\mathrm{j}} \leq \mathrm{c}$. There are two states for $\mathrm{v}_{\mathrm{j}}^{\mathrm{min}}$ that include the attractive $\left(\mathrm{q}_{\mathrm{j}} \varphi_{\mathrm{d}}<0\right)$ and repulsive $\left(\mathrm{q}_{\mathrm{j}} \varphi_{\mathrm{d}}>0\right)$ potentials. In an attractive potential, the plasma particle and the dust grain attract each other, and then, the initial velocity is not required for the incident particles. However, in repulsive potential, the plasma particle and the dust grain repel each other, and hence, the existence of an initial velocity $v_{j}^{\mathrm{min}}$ is necessary. Thus, in this case, $\mathrm{v}_{j}^{\mathrm{min}}$ becomes

$$
v_{j}^{\text {min }}=c \sqrt{1-\left(\frac{m_{j} c^{2}}{q_{j} \varphi_{d}}\right)^{2}} .
$$

Equation (9) can be calculated using integration by parts, incomplete beta function and hyper geometric functions, ${ }^{49,50}$ and combination methods.

Since ions are much heavier than electrons, initially the ion current is significantly less than electric current, and dust particles cause a negative charge. A little later, the ion current increases to the grain. Ultimately, both the ions and the electrical current are equal, which is an interesting situation for our study. The calculation for the case of a repulsive potential $\left(\mathrm{q}_{\mathrm{j}} \varphi_{\mathrm{d}}>0\right)$ is as follows:

$$
I_{e}=-r_{d}^{2} n_{e} G_{e}\left[1+H_{e} u_{e}^{2}\right],
$$

where $G_{e}$ and $H_{e}$ are, respectively, as

$$
\begin{aligned}
& G_{e}=P_{e} E_{e}-P_{e}^{\prime \prime} E_{e}^{\prime \prime}, \\
& H_{e}=\frac{P_{e}^{\prime \prime} E_{e}^{\prime \prime}-2 P_{e}^{\prime} E_{e}^{\prime}}{G_{e}},
\end{aligned}
$$

where

$$
\begin{gathered}
P_{e}=(-1)^{\theta_{e}}\left(\frac{1}{S_{e}}\right)^{-2}\left(\frac{\pi e c}{m_{e} \lambda_{e}}\right), \\
P_{e}^{\prime}=(-1)^{\theta_{e}}\left(\frac{1}{S_{e}}\right)^{-3}\left(\frac{\pi e c}{m_{e} \lambda_{e}}\right), \\
P_{e}^{\prime \prime}=(-1)^{\theta_{e}}\left(\frac{1}{S_{e}}\right)^{-4}\left(\frac{\pi e c}{m_{e} \lambda_{e}}\right), \\
E_{e}=\left[\frac{\theta_{e} !\left(1-\theta_{e}\right) !}{2 !}-\frac{1}{1+\theta_{e}}\left(\sum_{n=0}^{\infty} \frac{1+\theta_{e}}{1+\theta_{e}+n} \frac{\left(\theta_{e}+n-2\right) !}{\left(\theta_{e}-2\right) !} \frac{1}{n !}\right)\right], \\
E_{e}^{\prime}=\left[\frac{\theta_{e} !\left(2-\theta_{e}\right) !}{3 !}-\frac{1}{1+\theta_{e}}\left(\sum_{n=0}^{\infty} \frac{1+\theta_{e}}{1+\theta_{e}+n} \frac{\left(\theta_{e}+n-3\right) !}{\left(\theta_{e}-3\right) !} \frac{1}{n !}\right)\right], \\
E_{e}^{\prime \prime}=\left[\frac{\theta_{e} !\left(3-\theta_{e}\right) !}{4 !}-\frac{1}{1+\theta_{e}}\left(\sum_{n=0}^{\infty} \frac{1+\theta_{e}}{1+\theta_{e}+n} \frac{\left(\theta_{e}+n-4\right) !}{\left(\theta_{e}-4\right) !} \frac{1}{n !}\right)\right],
\end{gathered}
$$

and also 


$$
\begin{gathered}
\theta_{e}=\frac{1}{q_{e}-1}, \\
S_{e}=\alpha_{e}\left(q_{e}-1\right) .
\end{gathered}
$$

That $\lambda_{\mathrm{e}}$ is calculated by solving Eq. (7) as

$$
\lambda_{e}=\frac{1}{2 S_{e}} \sum_{n=0}^{\infty} \frac{\left(n-\theta_{e}-1\right) !}{\left(-\theta_{e}-1\right) !}\left[\frac{(n+2)(n-1)}{3 S_{e}^{2}}-1\right] .
$$

In an of attractive potential $\left(\mathrm{q}_{\mathrm{j}} \varphi_{\mathrm{d}}<0\right)$, however, the procedure for integration is similar approximate to Eq. (5), and the current is obtained as

$$
I_{i}=r_{d}^{2} n_{i} G_{i}\left[1+H_{i} u_{i}^{2}\right],
$$

where $G_{i}$ and $H_{i}$ are, respectively, given as

$$
\begin{aligned}
G_{i} & =P_{i} E_{i}-P_{i}^{\prime \prime} E_{i}^{\prime \prime}, \\
H_{i} & =\frac{P_{i}^{\prime \prime} E_{i}^{\prime \prime}-2 P_{i}^{\prime} E_{i}^{\prime}}{G_{i}},
\end{aligned}
$$

and in these

$$
\begin{gathered}
P_{i}=(-1)^{\theta_{i}}\left(\frac{1}{S_{i}}\right)^{-2}\left(\frac{\pi e c}{m_{i} \lambda_{i}}\right), \\
P_{i}^{\prime}=(-1)^{\theta_{i}}\left(\frac{1}{S_{i}}\right)^{-3}\left(\frac{\pi e c}{m_{i} \lambda_{i}}\right), \\
P_{i}^{\prime \prime}=(-1)^{\theta_{i}}\left(\frac{1}{S_{i}}\right)^{-4}\left(\frac{\pi e c}{m_{i} \lambda_{i}}\right), \\
E_{i}=\left[\frac{\theta_{i} !\left(1-\theta_{i}\right) !}{2 !}-\frac{1}{1+\theta_{i}}\left(\sum_{n=0}^{\infty} \frac{1+\theta_{i}}{1+\theta_{i}+n} \frac{\left(\theta_{i}+n-2\right) !}{\left(\theta_{i}-2\right) !} \frac{1}{n !}\right)\right], \\
E_{i}^{\prime}=\left[\frac{\theta_{i} !\left(2-\theta_{i}\right) !}{3 !}-\frac{1}{1+\theta_{i}}\left(\sum_{n=0}^{\infty} \frac{1+\theta_{i}}{1+\theta_{i}+n} \frac{\left(\theta_{i}+n-3\right) !}{\left(\theta_{i}-3\right) !} \frac{1}{n !}\right)\right], \\
E_{i}^{\prime \prime}=\left[\frac{\theta_{i} !\left(3-\theta_{i}\right) !}{4 !}-\frac{1}{1+\theta_{i}}\left(\sum_{n=0}^{\infty} \frac{1+\theta_{i}}{1+\theta_{i}+n} \frac{\left(\theta_{i}+n-4\right) !}{\left(\theta_{i}-4\right) !} \frac{1}{n !}\right)\right],
\end{gathered}
$$

and also

$$
\begin{gathered}
\theta_{i}=\frac{1}{q_{i}-1}, \\
S_{i}=\alpha_{i}\left(q_{i}-1\right) .
\end{gathered}
$$

That $\lambda_{\mathrm{i}}$ is calculated by solving Eq. (7) as

$$
\lambda_{i}=\frac{1}{2 S_{i}} \sum_{n=0}^{\infty} \frac{\left(n-\theta_{i}-1\right) !}{\left(-\theta_{i}-1\right) !}\left[\frac{(n+2)(n-1)}{3 S_{i}^{2}}-1\right] .
$$

We assume that the system is neutral from almost an electrical point of view. The quasineutrality condition of the system is as follows:

$$
\frac{n_{e}}{n_{i}}=1-Z_{d} \frac{n_{d}}{n_{i}},
$$

where $n_{d}$ and $Z_{d}$ are the density and the number of charges of the dust grain, respectively. When $\mathrm{Z}_{\mathrm{d}} \mathrm{n}_{\mathrm{d}} / \mathrm{n}_{\mathrm{i}} \ll 1$, the dust particles can be assumed to be isolated, and when this ratio is comparable to unity, the seed can be assumed to be nonisolated. In nonisolated cases, increasing the density of the number of dust grains means that the seeds have a great deal of appetite for electrons, but the number of electrons in each dust grain is small dust. As noted above, the ratio $\mathrm{Z}_{\mathrm{d}} \mathrm{n}_{\mathrm{d}} / \mathrm{n}_{\mathrm{i}} \ll 1$ is an important factor in determining the dusty plasma behavior, and it is common in the literature that the parameter is defined to consider this ratio; it is in the form,

$$
P=-\frac{m_{e} c^{2} r_{d} n_{d}}{e^{2} n_{i}}
$$

that $\mathrm{P}=-\mathrm{Z}_{\mathrm{d}} \mathrm{n}_{\mathrm{d}} / \mathrm{n}_{\mathrm{i}} \mathrm{u}_{\mathrm{e}}$ is the normalized dust grain number density, and thus the $P$ parameter is equivalent with $Z_{d} n_{d} / n_{i}$ that play an important role in determining the dusty plasma behavior. Now, as the final step for evaluating the dust grain charging process and the electrical potential of dust grain, we have to use Eq. (11) for electrons and Eq. (23) for ions and owing to the fact that $I_{i}+I_{e}=0$, we can write

$$
\frac{G_{i}\left[1+H_{i} u_{i}^{2}\right]}{G_{e}\left[1+H_{e} u_{e}^{2}\right]}=1-P u_{e},
$$

where

$$
u_{i}=-\left(\frac{m_{e}}{m_{i}}\right) u_{e}
$$

To clarify this issue, now we have to solve Eq. (37). It is clear that this equation is very nonlinear and therefore has no analytical solutions. Thus, with boundary conditions and using numerical methods, the electric potential of the dust grain in plasma can be obtained in terms of the P parameter value. In order to consider the electrical potential behavior of dust grains in term of $\mathrm{P}$, a logarithmic form can be used to make the process of dust grain charging more clearly.

\section{RESULTS AND DISCUSSION}

From Eq. (37), it is determined that the electrical potential of dust grain can change under the dust grain charging process. This equation shows that the dust grain electrical potential in the relativistic regime is dependent on the nonextensivity degree of system and also the characteristics of the dusty plasma such as the density of grain dust, the particle size of the dust, electron and ion temperature, and plasma gas. In this section, the numerical solution of nonlinear Eq. (37) is obtained, and the effects of nonextensivity parameters $\mathrm{q}_{\mathrm{i}}$ and $\mathrm{q}_{\mathrm{e}}$, the ratio of temperature ion-to-electron and plasma gas on dust grain electrical potential in the relativistic regime is considered. In these figures, the particles with high relativistic energies are assumed to have a temperature of $100 \mathrm{GeV}$ electrons, and thus, the $\mathrm{P}$ parameter is considered. When a dust grain size is $1 \mu$, the $\mathrm{P}$ parameter is proportional to the ratio of dust to ion density. For example, the energy spectrum is power-law in supernova explosion, and thus the ion and dust density is $10^{19} \mathrm{~cm}^{-3}$ and $10^{19} \mathrm{~cm}^{-3}$, respectively. ${ }^{26,46,51}$ In Figs. 1(a)-1(c), the effect nonextensivity degree of the electron in the relativistic mode on the dust grain electrical potential for three of plasma gases is presented as a function of $\log P$. Plasmas are considered as hydrogen, helium, and carbon plasma gases as shown in Figs. 1(a)-1(c), respectively. Relativistic heavy plasma ions such as carbon exist in collapse of a star, 

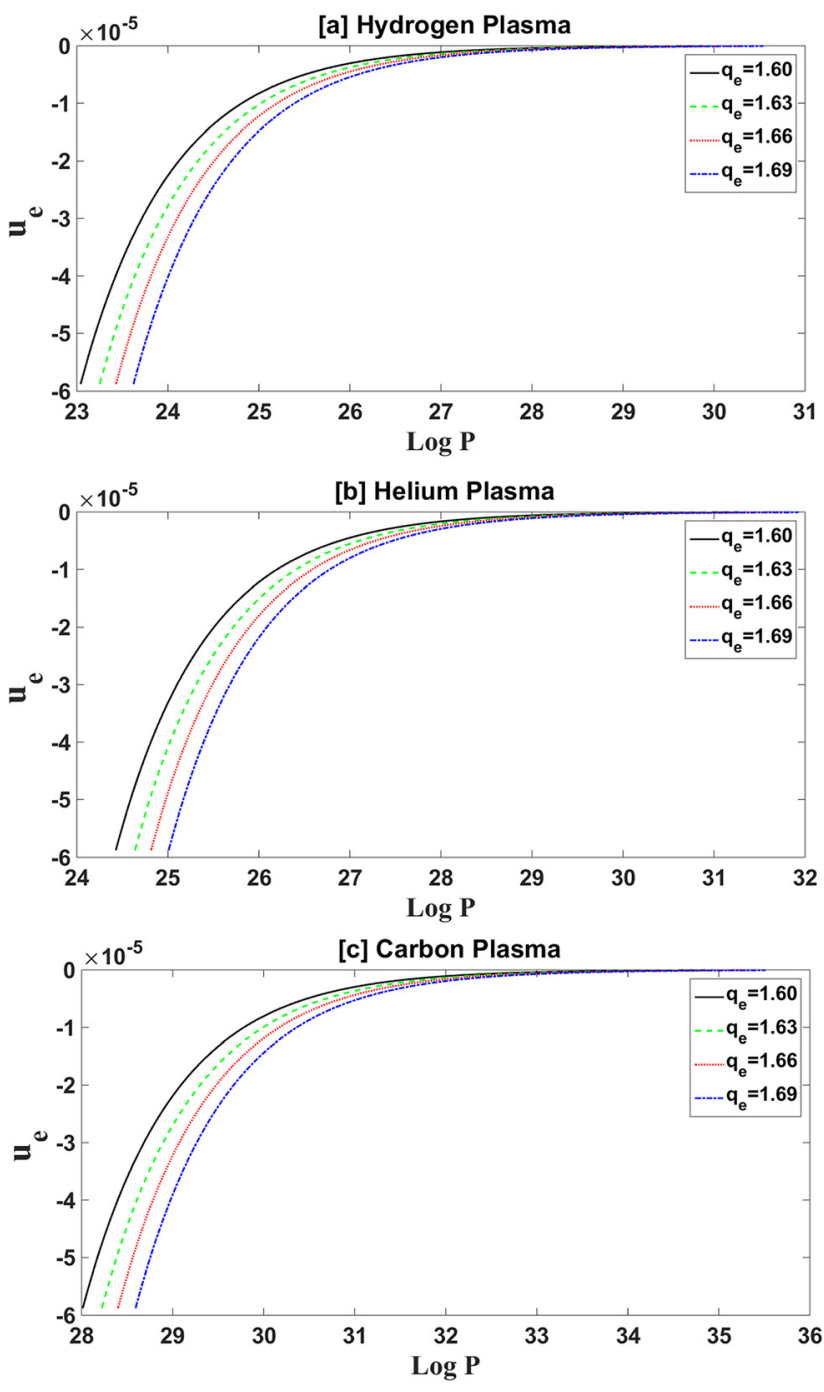

FIG. 1. The variations of the dust grain electrical potential vs $\log P$ with different nonextensivity of electron in the relativistic regime. (a) Hydrogen plasma, (b) helium plasma, and (c) carbon plasma that $q_{\mathrm{e}}=1.60$ (solid line), $q_{\mathrm{e}}=1.63$ (dashed line), $\mathrm{q}_{\mathrm{e}}=1.66$ (dotted line), and $\mathrm{q}_{\mathrm{e}}=1.69$ (dash dotted line) with nonextensivity degree of ion $\mathrm{q}_{i}=0.9$, and the plasma is isothermal $\left(\mathrm{T}_{\mathrm{e}}=\mathrm{T}_{\mathrm{i}}\right)$.

supernova explosions, beam carbon ion in laser-solid interactions, and formation of hypernuclei in relativistic ion collisions for nuclear/particle physics and astrophysics. ${ }^{52-55}$ They exist in explosive emission when the field enhancement factor for the carbon cathode is about $\beta=100$, and applied voltage is $2 \mathrm{MV}$, and thus, carbon velocity is about $10^{7} \mathrm{~m} / \mathrm{s}{ }^{56}$ These curves are plotted for the different values of $\mathrm{q}_{\mathrm{e}}=1.60$ (solid line), $\mathrm{q}_{\mathrm{e}}=1.63$ (dashed line), $\mathrm{q}_{\mathrm{e}}=1.66$ (dotted line), and $\mathrm{q}_{\mathrm{e}}=1.69$ (dash dotted line) in the relativistic regime when nonextensivity degree of ion $\mathrm{q}_{\mathrm{i}}=0.9$, and the plasma is isothermal $\left(T_{e}=T_{i}\right)$. We find that the degree of nonextensivity of electron has almost no any effect on the dust grain electrical potential when $\mathrm{P}$ is increased, but by decreasing degree of nonextensivity of electron, it has the significant effect on the relationship between $\mathrm{u}_{\mathrm{e}}$ and P. Moreover, by increasing the degree of nonextensivity of electron, $\mathrm{P}$ is increased, and larger electrical potential are obtained at higher amounts of P. In Fig. 1, the variations of the dust grain electrical potential vs the dust density is shown if it is assumed that the ion density and dust grain radius to be constant. Then by increasing the degree of electron nonextensivity, the bigger dust grain electrical potential is available for the bigger dust density. As another result, it is indicated that the value of $\mathrm{u}_{\mathrm{e}}$ decreases in the relativistic regime, and as equivalent, the average of dust grain charge decreases. Also, as it can be seen from Fig. 1 that there is a critical region in which the electrical potential of the dust grains, and by increasing of the dust density, dust particles will be seen as a component from ordinary multi-ionic plasma that shows collective behavior. By comparing these figures that are plotted in the relativistic regime with results of Ref. 45 in the nonrelativistic regime, it is indicated that in the relativistic regime, the value of $\mathrm{P}$ is limited, and the electrical potential of the dust grain varies, whereas in the nonrelativistic regime, the value of $\mathrm{P}$ is not limited, and the dust grain electrical potential tends to be constant in small amounts of $\mathrm{P}$. Furthermore, the region of critical in the relativistic regime is very smaller than that in the nonrelativistic regime, and the transition to zero for the electrical potential of dust grain is much faster. By comparing the results of Ref. 45 and 48 and the relativistic regime, when we assume that the ion density is constant, it is not possible to dust charging for values of low dust densities. In addition, according to these figures, we also find that by changing the plasma gas when the ions are heavy, the amount of $\mathrm{P}$ increases. This means that if the density of ions and dust grain size is constant, when the heavier the plasma gas is, the most of electrical potential of the dust grain is in larger amounts of dust density than before. It is worth noting that by calculating the area under the curve, the relation Area $=1-n_{e} / n_{i}$ is obtained, and then, the area under the curve represents the relationship between electrons density ratio of ions. Thus, Fig. 1 shows that as the $\mathrm{q}_{\mathrm{e}}$ parameter increases, the area under the curve also decreases, resulting in an increase in the electron-ion density ratio. In other words, at higher degree of electron nonextensivity, the role of electrons is larger than ions.

Figures 2(a)-2(c) show the variations of the electrical potential of dust grain vs $\log \mathrm{P}$ for different values ion nonextensivity degree in hydrogen, helium, and carbon plasmas gases, respectively. In these figures, the curves are plotted for the different nonextensivity of ion in the relativistic regime that $\mathrm{q}_{\mathrm{i}}=0.9110$ (solid line), $\mathrm{q}_{\mathrm{i}}=0.9115$ (dashed line), $\mathrm{q}_{\mathrm{i}}=0.9120$ (dotted line) and $\mathrm{q}_{\mathrm{i}}=0.9125$ (dash dotted line). In these figures, nonextensivity degree of electron $\mathrm{q}_{\mathrm{e}}=1.6$, and the plasma is isothermal too. If it is assumed that dust density and dust grain size are constant, from Fig. 2 it indicates that as ion nonextensivity degree rises, bigger electrical potential for dust grain occurs at plasmas with higher ion density. On the other hand, when the ion density and dust grain size are constant, by increasing the ion nonextensivity degree, the bigger electrical potential of dust grain is obtained for higher dust density. This behavior is in contrast to Fig. 1, and this indicates that the increase in $\mathrm{q}_{e}$ is directly related to the decrease in $\mathrm{q}_{\mathrm{i}}$. Furthermore, it is found that the difference between the $\mathrm{P}$ values of carbon, helium, and hydrogen plasmas increases slightly when the degree of nonextensivity of ion increases. It can be seen from Fig. 2 that as the $q_{i}$ parameter increases, the surface area of the curved laser increases and the electron to ion density ratio decreases. In other words, at higher parameters of ions, the role of ions is larger than that 

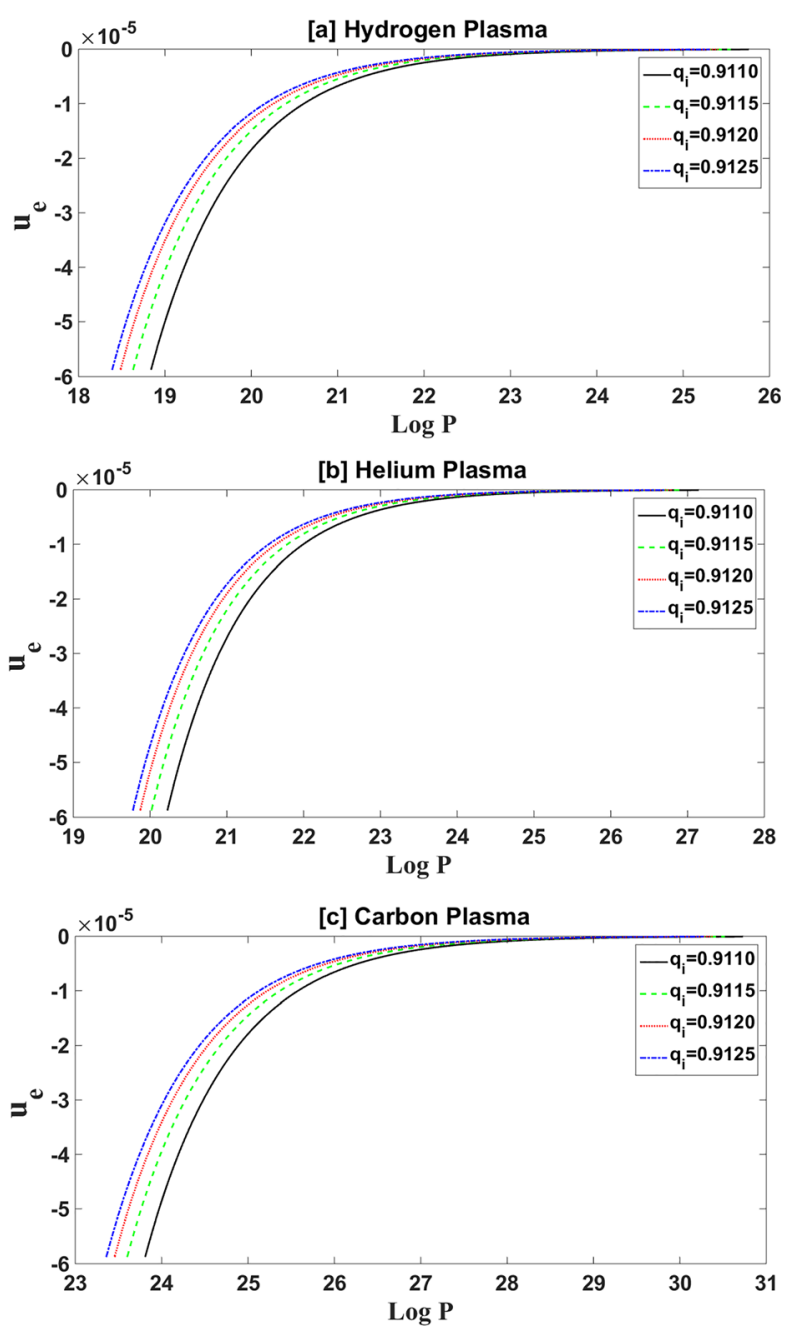

FIG. 2. The variations of the dust grain electrical potential vs $\log P$ with different nonextensivity of ion in the relativistic regime (a) hydrogen plasma, (b) helium plasma, and (c) carbon plasma that $\mathrm{q}_{\mathrm{i}}=0.9110$ (solid line), $\mathrm{q}_{\mathrm{i}}=0.9115$ (dashed line), $q_{i}=0.9120$ (dotted line) and $q_{i}=0.9125$ (dash dotted line) with nonextensivity degree of electron $q_{e}=1.6$, and the plasma is isothermal $\left(T_{e}=T_{i}\right)$.

of electrons. By comparing the results as shown in Figs. 1 and 2, it is clear that the $\mathrm{q}_{\mathrm{e}}$ electron parameter has the most influence on the electrons and at the higher electron densities, and also the $\mathrm{q}_{\mathrm{i}}$ ion parameter has the most effect on the ions and at the higher ion densities, and then, the density of spaces has the most influence on degree of nonextensivity.

The influence of plasma gases on the dust grain electrical potential is presented as a function of $\log P$ in Fig. 3 that the curves are plotted for three of plasma gases including hydrogen plasma (solid line), helium plasma (dashed line), and carbon plasma (dash dotted line). The results are obtained for a plasma isothermal with degree of nonextensivity 1.6 and 0.9 for the electron and the ion, respectively. By comparing curves, it is obvious that as mass of ion increases, the dust grain electrical potential decreases. It is obvious that if the temperature and the resultant energy are constant, then due to the mass-energy

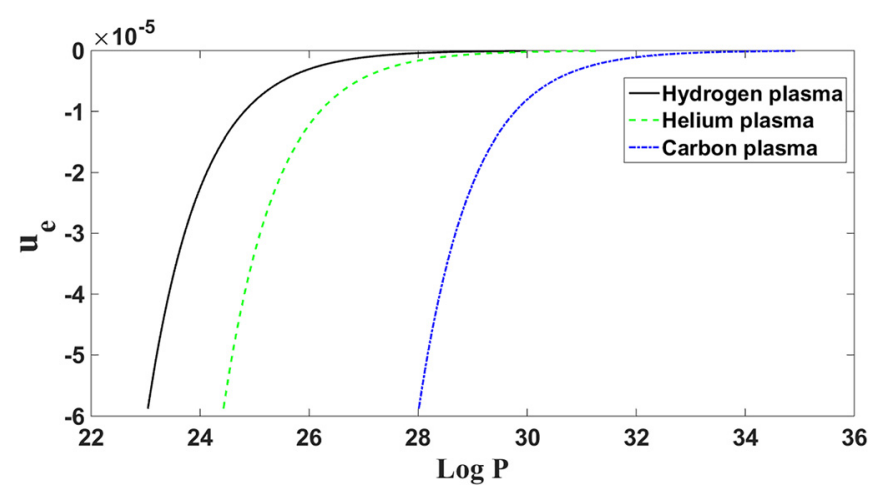

FIG. 3. The relationship between dust grain electrical potential and $\log P$ for different kinds of plasma gases in temperature ratios $T_{i} / T_{e}=1$ in the relativistic regime that hydrogen plasma (solid line), helium plasma (dashed line), and carbon plasma (dash dotted line) with nonextensivity degree $q_{i}=0.9$ and $q_{e}=1.6$.

equivalence relation $\mathrm{E}=\gamma \mathrm{m}_{0} \mathrm{c}^{2}$ (that $\mathrm{m}_{0}=\mathrm{m}_{\mathrm{i}}$ is rest mass of ion), and the mass of the ions increases, the $\gamma=\left(1-\beta^{2}\right)^{-1 / 2}$ value must also decreases, and the $\gamma$ gain indicates that $\beta$ and the ion velocity decrease. In other words, increasing the mass of ions reduces the effects of relativistic, and then, a decrease in the relativistic effects acts as a reduction in the dust grain electrical potential. This means that for the specific dust density values (identical P), the electric potential of the dust grain in light gases is smaller or in the weak relativistic regime, and the dust grain electrical potential is higher. It is shown that by increasing mass of ions, the minimum possible value for the $\mathrm{P}$ increases, and the horizontal axis shifted forward. In addition, it is clear from the figures that the difference between the curves of the dust grain electrical potential changes for the hydrogen and helium plasma is much greater than for the carbon plasma. This indicates that the heavier the plasma gas, the variations in the dust grain electrical potential curve vs $\mathrm{P}$ are smaller, and the role of the mass of the ions becomes smaller. In other words, in light plasma, the mass effect of ions is much greater on the electrical potential of the dust grain than that in heavier plasma. On the other hand, the results show that as the plasma becomes heavier, the area under the curve decreases, and as a result, the electron to ion density ratio increases; it is clear that in this situation, the role of electrons in plasma is more pronounced than the role of ions.

Figures 4(a) and 4(b) show the variations of the electrical potential of dust grain in terms of $\log P$ in different values of ion-to-electron temperature ratios for smaller and larger than unity, respectively. In these figures, the scales are the same for easier comparison, and curves also are plotted for the different temperature ratios of ion-to-electron in (a) that $\mathrm{T}_{\mathrm{i}} / \mathrm{T}_{\mathrm{e}}=0.7$ (solid line), $\mathrm{T}_{\mathrm{i}} / \mathrm{T}_{\mathrm{e}}=0.8$ (dashed line), $\mathrm{T}_{\mathrm{i}} / \mathrm{T}_{\mathrm{e}}$ $=0.9$ (dotted line) and $\mathrm{T}_{\mathrm{i}} / \mathrm{T}_{\mathrm{e}}=1$ (dash dotted line) and in (b) that $\mathrm{T}_{\mathrm{i}} / \mathrm{T}_{\mathrm{e}}=1.1$ (solid line), $\mathrm{T}_{\mathrm{i}} / \mathrm{T}_{\mathrm{e}}=1.2$ (dashed line), $\mathrm{T}_{\mathrm{i}} / \mathrm{T}_{\mathrm{e}}=1.3$ (dotted line), and $\mathrm{T}_{\mathrm{i}} / \mathrm{T}_{\mathrm{e}}=1.4$ (dash dotted line). Temperature ratios higher than one, for example, exist in the magnetosheath where magnetosheath particles are solar wind particles energized by the bow shock, and the bow shock energy ions more than electrons, therefore, in the magnetosheath, the plasma sheet, and the magnetotail $\mathrm{Ti} / \mathrm{Te}$ can be greater than one. ${ }^{57-59}$ It is shown in Fig. 4 that an enhancement in ion temperature leads to a smaller dust grain electrical potential. It is obvious that a temperature ratio 

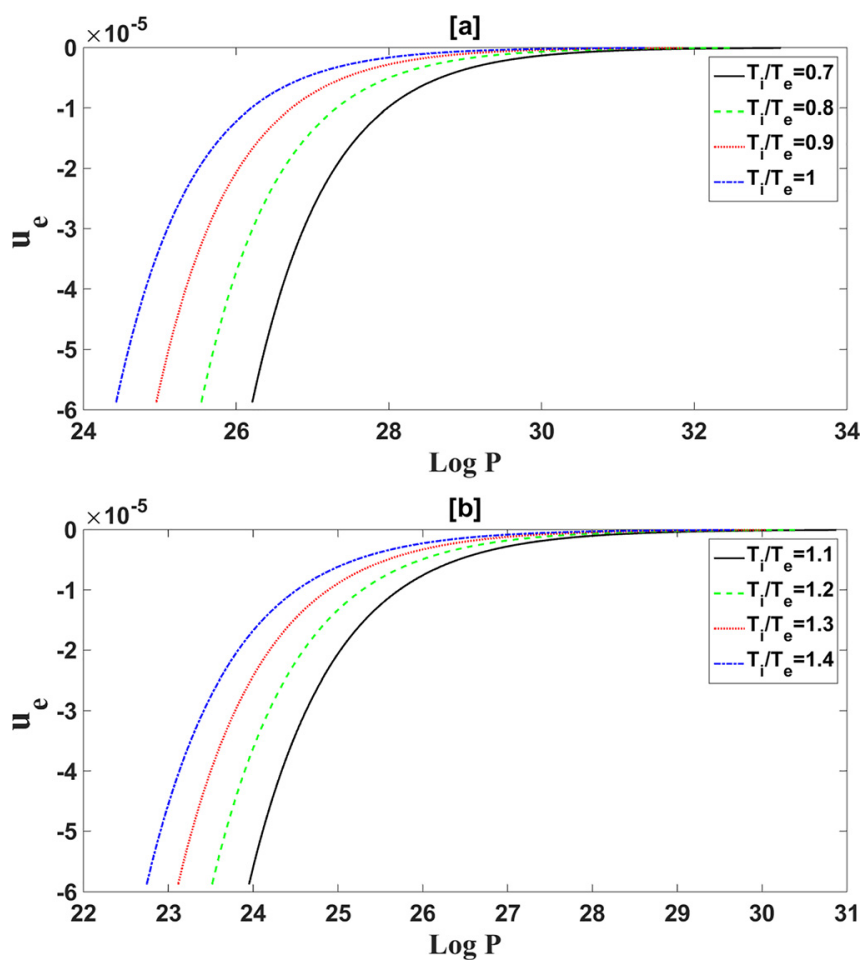

FIG. 4. The relationship between dust grain electrical potential and log $P$ for different values of temperature ratios of ion-to-electron (a) smaller and (b) larger than unity in the relativistic regime that in (a) $T_{i} / T_{e}=0.7$ (solid line), $T_{i} / T_{e}=0.8$ (dashed line), $T_{i} / T_{e}=0.9$ (dotted line), and $T_{i} / T_{e}=1$ (dash dotted line) and in (b) $T_{i} / T_{e}=1.1$ (solid line), $T_{i} / T_{e}=1.2$ (dashed line), $T_{i} / T_{e}=1.3$ (dotted line), and $T_{i} / T_{e}=1.4$ (dash dotted line) with nonextensivity degree $q_{i}=0.9$ and $q_{e}=1.6$ in helium and isothermal plasma.

of ion-to-electron smaller leads to a bigger electrical potential of dust grain too. The physical reason is that as the ionic temperature raises, the velocity of the ions in the relativistic regime increases as a result of Eq. (3), the collision cross section, and thus, the ion current of the dust grain charging processes decrease. As a result of the static state assumption, the reduction of ionic current leads to a decrease in the electron current, so the charge of dust grain and its electrical potential decrease. An important point in these figures is that by increasing ion temperature when it is larger than one, the curves of dust grain electrical potential are close together, but in smaller than one, the curves of dust grain electrical potential are far from together. In other words, at ion temperatures above the electron temperature, the effect of temperature on the electrical potential variations of the dust grain will be less pronounced, but when the temperature of electrons are larger than the ions, the temperature has a greater effect on the dust grain electrical potential. As the temperature of the ions increases, the range available for the amount of $\mathrm{P}$ or density of dust particles increases. Moreover, it can be seen from Fig. 4 that as the temperature of the ion increases, the area under the curve decreases, resulting in a decrease in the electron-ion density ratio. In other words, the role of ions against electrons in plasma increases.

\section{SUMMARY AND CONCLUSION}

In summary, with the help of the OLM theory and a kinetic model in the relativistic regime, we considered the dust grain charging process using the relativistic cross section. The dust grain electrical potential was calculated by using the relativistic Tsallis distribution function in the dust grain charging process, and finally, the role of different parameters on the dust grain electrical potential was considered by numerical analyses of Eq. (37). We found that decreasing the degree of electron nonextensivity has a significant effect on the relation between $\mathrm{u}_{\mathrm{e}}$ and $\mathrm{P}$ and the greater the amount of $\mathrm{P}$, the greater the electrical potential of the dust grain. By comparing the results of this work in the relativistic regime and results of Ref. 45 in the nonrelativistic regime, it indicated that in the relativistic regime, the value of $\mathrm{P}$ was limited, and the electrical potential of dust grains was always variable, whereas in the nonrelativistic regime, the value of $\mathrm{P}$ was not limited, and the dust grain electrical potential tended to be constant in small amounts of $\mathrm{P}$. As another result, it indicated that there was a critical region for the electrical potential of the dust grains and by increasing the dust density, dust particles were seen as a component from an ordinary multi-ionic plasma that showed collective behavior. Also, the critical region in the relativistic regime was much smaller than the nonrelativistic regime and the transition to zero for the electrical potential of the dust grain was much faster. In addition, we found when the ions were heavy, the amount of $\mathrm{P}$ increased. It was shown that as the degree of ion nonextensivity rises, there is a bigger electrical potential for dust grains to occur at plasmas in higher ion density and by increasing the degree of ion nonextensivity, there is a bigger electrical potential of dust grains in lower dust density. Moreover, we indicated that the behavior of nonextensivity was in contrast to ion and electron, and it was found that the density of species in plasma has the most influence on the degree of nonextensivity. It indicated that by the increasing mass of ion, the dust grain electrical potential and the relativistic effects decreased and also by increasing mass of ions, the amount of $\mathrm{P}$ was shifted forward. In addition, this indicated that in light plasma, the mass effect of ions was much greater on the electrical potential of the dust grain than heavy plasma. On the other hand, the results showed that as the plasma became heavier, the role of electrons in plasma was more pronounced than the role of ions. It was obvious that a temperature ratio of smaller ion-to-electron lead to a bigger electrical potential of dust grain too. Moreover, it was seen that as the temperature of the ions increased, the role of ions against electrons in plasma increased. Finally, when the temperature of the ion was higher than the electron temperature, the effect of temperature on the electrical potential variations of the dust grain was less pronounced, but when the temperature of electrons was larger than the ions, the temperature had a greater effect on the dust grain electrical potential.

\section{REFERENCES}

${ }^{1}$ K. Huang, Statistical Physics, 1st ed. (CRC Press, 2001).

${ }^{2}$ C. Beck, G. Benede, A. Rapisarda, and C. Tsallis, Complexity, Metastability and Nonextensivity (World Scientific Press, 2004).

${ }^{3}$ C. Tsallis, "Possible generalization of Boltzmann-Gibbs statistics," J. Stat. Phys. 52, 479-487 (1988).

${ }^{4}$ E. Vladimir and E. Gregor, Complex and Dusty Plasmas: From Laboratory to Space, 1st ed. (CRC Press, 2009).

${ }^{5}$ C. Tsallis, D. Prato, and A. R. Plastino, "Nonextensive statistical mechanics: Some links with astronomical phenomena," Astrophys. Space Sci. 290(3-4), 259-274 (2004). 
${ }^{6} \mathrm{R}$. Guo and J. Du, "Are power law distributions an equilibrium distribution or a stationary one equilibrium distribution?," Physica A 406, 281-286 (2014).

${ }^{7}$ A. Barkan, N. D'Angelo, and R. L. Merlino, "Charging of dust grains in a plasma," Phys. Rev. Lett. 73(23), 3093-3096 (1994).

${ }^{8} \mathrm{M}$. Swisdak, "The generation of random variates from a relativistic Maxwellian distribution," Phys. Plasmas 20, 062110 (2013).

${ }^{9} \mathrm{~L}$. Guo and J. Du, "Thermodynamic potentials and thermodynamic relations in nonextensive thermodynamics," Physica A 390, 183-188 (2011).

${ }^{10} \mathrm{H}$. Yu and $\mathrm{j} \mathrm{Du}$, "The nonextensive parameter for nonequilibrium electron gas in an electromagnetic field," Ann. Phys. 350, 302-309 (2014).

"IJ. M. Liu, J. S. De Groot, J. P. Matte, T. W. Johnston, and R. P. Drake, "Measurements Bremsstrahlung inverse absorption and non-Maxwellian electron velocity distributions," Phys. Rev. Lett. 72(17), 2717-2720 (1994).

${ }^{12}$ D. A. Moreira, E. L. Albuquerque, L. R. Silva, D. S. Galvão, and D. A. Moreira, "Low-temperature specific heat spectra considering nonextensive long-range correlated quasiperiodic DNA molecules," Physica A 387, 5477-5482 (2008)

${ }^{13}$ S. I. Krasheninnikov, R. D. Smirnov, and D. L. Rudakov, "Dust in magnetic fusion devices," Plasma Phys. Controlled Fusion 53(8), 083001 (2011).

${ }^{14} \mathrm{R}$. Mendis and M. Rosenberg, "Cosmic dusty plasma," Annu. Rev. Astron. Astrophys. 32(1), 419-463 (1994).

${ }^{15} \mathrm{~A}$. Valentin and R. Kartinov, "Skeletal structures in the images of cosmic dust clouds and solar system planets," IEEE Trans. Plasma Sci. 35(4), 767 (2007).

${ }^{16}$ Y. L. Alpert, A. V. Gurevich, and L. P. Pitaevskii, "Space physics with artificial satellites," Am. J. Phys. 34(6), 544-544 (1966).

${ }^{17}$ H. Saleem, W. M. Moslem, and P. K. Shukla, "Solar wind interactions with the dusty magnetosphere of Jupiter produce shocks and solitons associated with nonlinear drift wave," J. Geophys. Res. 117, 08220, https://doi.org/10.1029/ 2011JA017306 (2012).

${ }^{18} \mathrm{G}$. Rostoker, "Recent advances in the study of electric and magnetic fields in the ionosphere and magnetosphere," J. Geomagn. Geoelectr. 32, 431-470 (1980).

${ }^{19}$ R. Silva, J. S. Alcaniz, and J. A. S. Lima, "Constraining nonextensive statistics with plasma oscillation data," Physica A 356, 509 (2005).

${ }^{20} \mathrm{~F}$. Caruso, A. Pluchino, V. Latora, S. Vinciguerra, and A. Rapisarda, "Analysis of self-organized criticality in the Olami-Feder-Christensen model and in real earthquakes," Phys. Rev. E: Stat., Nonlinear, Soft Matter Phys. 75(5 Pt 2), 055101 (2007).

${ }^{21}$ A. R. Plastino and A. Plastino, "Stellar polytropes and Tsallis' entropy," Phys. Lett. A. 174, 384-386 (1993).

${ }^{22}$ A. Y. Wong, N. D. Angelo, and R. W. Motley, "Propagation and damping of ion acoustic waves in highly ionized plasmas," Phys. Rev. Lett. 9(10), 415-416 (1962).

${ }^{23}$ J. A. S. Lima, R. Silva, Jr., and J. Santos, "Plasma oscillations and nonextensive statistic," Phys. Rev. E 61(3), 3260-3263 (2000).

${ }^{24} \mathrm{~B}$. M. Boghosian, "Thermodynamic description of the relaxation of twodimensional turbulence using Tsallis statistics," Phys. Rev. E 53, 4754-4763 (1996).

${ }^{25}$ G. Kaniadakis, A. Lavagno, and F. Quarati, "Generalized statistics and solar neutrinos," Phys. Lett. B 369, 308-312 (1996).

${ }^{26}$ M. E. Dieckmann, P. K. Shukla, and B. Eliasson, "Formation of electrostatic structures by wakefield acceleration in ultrarelativistic plasma flows: Electron acceleration to cosmic ray energies," Phys. Plasmas 13, 062905-062908 (2006).

${ }^{27}$ J. J. Podesta, "Landau damping in relativistic plasmas with power-law distributions and applications to solar wind electrons," Phys. Plasmas 15, 122902-122910 (2008).

${ }^{28}$ J. Kumari and R. S. Pandey, "Study of VLF wave with relativistic effect in Saturn magnetosphere in the presence of parallel A.C. electric field," Adv. Space Res. 63(7), 2279-2289 (2019).

${ }^{29}$ I. Lourek and M. Tribeche, "Dust charging current in non equilibrium dusty plasma in the context of Kaniadakis generalization," Physica A 517, 522-529 (2019).

${ }^{30}$ A. Hasegawa, K. Mima, and M. Duong-van, "Plasma distribution function in a superthermal radiation field," Phys. Rev. Lett. 54(24), 2608-2610 (1985).
${ }^{31}$ S. Q. LIU and X. C. CHEN, "Dispersion relation of transverse oscillation in relativistic plasmas with non-extensive distribution,” J. Plasma Phys. 77(5), 653-662 (2011).

${ }^{32} \mathrm{G}$. Lina, J. Du, and Z. Liu, "The property of $\kappa$-deformed statistics for a relativistic gas in an electromagnetic field: $\kappa$ parameter and $\kappa$-distribution," Phys. Lett. A. 367, 431-435 (2007).

${ }^{33}$ V. H. Hamity and D. E. Barraco, "Generalized nonextensive thermodynamics applied to the cosmic background radiation in a Robertson-Walker universe," Phys. Rev. Lett. 76(25), 4664-4666 (1996).

${ }^{34}$ A. R. Plastino, A. Plastino, and H. Vucetich, "A quantitative test of Gibbs' statistical mechanics,” Phys. Lett. A 207(1-2), 42-46 (1995).

${ }^{35}$ C. Tsallis, F. C. Sa Barreto, and E. D. Loh, "Generalization of the Planck radiation law and application to the cosmic microwave background radiation," Phys. Rev. E 52(2), 1447-1451 (1995).

${ }^{36}$ A. Lavagno, G. Kaniadakis, M. Rego-Monteiro, P. Quarati, and C. Tsallis, "Non-extensive thermostatistical approach of the peculiar velocity function of galaxy clusters," Astrophys. Lett. Commun. 35, 449-451 (1998).

${ }^{37} \mathrm{C}$. Tsallis, "Entropic nonextensivity: A possible measure of complexity," Chaos, Solitions Fractals 13, 371-391 (2002).

${ }^{38} \mathrm{H}$. M. Mott-Smith and I. Langmuir, "The theory of collectors in gaseous discharges," Phys. Rev. 28(4), 727-763 (1926).

${ }^{39}$ J. G. Laframboise, "Theory of spherical and cylindrical Langmuir probes in a collisionless, Maxwellian plasma at rest," DTIC Document, UTIAS Report No. $100,1966$.

${ }^{40}$ O. Havnes, C. K. Goertz, G. E. Morfill, E. Grün, and W. Ip, "Dust charges, cloud potential, and instabilities in a dust cloud embedded in a plasma," J. Geophysical Res.: Space Phys. 92(A3), 2281-2287 (1987).

${ }^{41}$ R. V. Kennedy and J. E. Allen, "The floating potential of spherical probes and dust grains. II: Orbit motion theory," J. Plasma Phys. 69(6), 485-506 (2003).

${ }^{42} \mathrm{M}$. Salimullah, I. Sandberg, and P. K. Shukla, "Dust charge fluctuations in a magnetized dusty plasma," Phys. Rev. E 68, 027403 (2003).

${ }^{43} \mathrm{C}$. T. N. Willis, M. Coppins, M. Bacharis, and J. E. Allen, "The effect of dust grain size on the floating potential of dust in a collisionless plasma," Plasma Sources Sci. Technol. 19(6), 065022 (2010).

${ }^{44} \mathrm{M}$. Lampe, "Limits of validity for orbit-motion-limited theory for a small floating collector," J. Plasma Phys. 65(3), 171-180 (2001).

${ }^{45} \mathrm{~J}$. Gong and J. Du, "Dust charging processes in the nonequilibrium dusty plasma with nonextensive power-law distribution," Phys. Plasmas 19, 023704-6 (2012).

${ }^{46} \mathrm{R}$. Bingham and V. N. Tsytovich, "Comments on relativistic dust particles forming the highest energy cosmic rays," Astroparticle Phys. 12, 35 (1999).

${ }^{47}$ A. Lavagno, "Relativistic nonextensive thermodynamics," Phys. Lett. A 301, 13-18 (2002).

${ }^{48}$ P. K. Shukla and A. A. Mamun, Introduction to Dusty Plasma Physics (IOP Publishing Ltd., 2002).

${ }^{49}$ A. Jeffrey and D. Z. Willinger, Table of Integrals, Series, and Products (Elsevier Science, 2000).

${ }^{50}$ S. Gradshteyn and I. M. Ryzhik, Table of Integrals, Series, and Products, 7 th ed. (Academic Press, 2007).

${ }^{51}$ S. I. Popel and A. A. Gisko, "Charged dust and shock phenomena in the solar system,” Nonlinear Processes Geophys. 13, 223-229 (2006).

${ }^{52}$ A. Botvina, M. Bleicher, and N. Buyukcizmeci, "Formation of hypernuclei in relativistic ion, hadron and lepton collisions," AIP Conf. Proc. 2130, 040007 (2019).

${ }^{53}$ T. J. Galama, P. M. Vreeswijk, J. van Paradijs, C. Kouveliotou, T. Augusteijn, H. Böhnhardt, J. P. Brewer, V. Doublier, J.-F. Gonzalez, B. Leibundgut, C. Lidman, O. R. Hainaut, F. Patat, J. Heise, J. in’t Zand, K. Hurley, P. J. Groot, R. G. Strom, P. A. Mazzali, K. Iwamoto, K. Nomoto, H. Umeda, T. Nakamura, T. R. Young, T. Suzuki, T. Shigeyama, T. Koshut, M. Kippen, C. Robinson, P. de Wildt, R. A. M. J. Wijers, N. Tanvir, J. Greiner, E. Pian, E. Palazzi, F. Frontera, N. Masetti, L. Nicastro, M. Feroci, E. Costa, L. Piro, B. A. Peterson, C. Tinney, B. Boyle, R. Cannon, R. Stathakis, E. Sadler, M. C. Begam, and P. Ianna, "An unusual supernova in the error box of the g-ray burst of 25 April 1998," Nature 395, 670 (1998). 
${ }^{54}$ D. Jung, B. J. Albright, L. Yin, D. C. Gautier, R. Shah, S. Palaniyappan, S. Letzring, B. Dromey, H.-C. Wu, T. Shimada, R. P. Johnson, M. Roth, J. C. Fernandez, D. Habs, and B. M. Hegelich, "Beam profiles of proton and carbon ions in the relativistic transparency regime," New J. Phys. 15, 123035 (2013).

${ }^{55}$ J. C. TAN, C. D. Matzner, and C. F. Mckee, "Trans-relativistic blast waves in supernovae as gamma-ray burst progenitors," The Astrophys. J. 551, 946 (2001).

${ }^{56}$ Y. Shen, L. Xia, H. Zhang, X. Liu, A. Yang, J. Shi, and L. Zhang, "Plasmainduced field emission study of carbon nanotube cathode," Phys. Rev. Spec. Top. - Accel. Beams 14, 104701 (2011).
${ }^{57}$ C. Wang, M. Gkioulidou, L. Lyons, and V. Angelopoulos, "Spatial distributions of the ion to electron temperature ratio in the magnetosheath and plasma sheet," J. Geophys. Res. 117, A08215, https://doi.org/10.1029/2012JA017658 (2012).

${ }^{58}$ W. Baumjohann, G. Paschmann, and C. A. Cattell, “Average plasma properties in the central plasma shee,” J. Geophys. Res. 94, 6597, https://doi.org/10.1029/ JA094iA06p06597 (1989).

${ }^{59}$ A. V. Artemyev, W. Baumjohann, A. A. Petrukovich, R. Nakamura, I. Dandouras, and A. Fazakerley, "Proton/electron temperature ratio in the magnetotail," Ann. Geophys. 29, 2253 (2011). 